\title{
Smart Energy: Herausforderungen an eine interdisziplinäre Zukunft
}

\section{G. Brauner OVE}

Online publiziert am 25. April 2015

(C) Springer Verlag Wien 2015

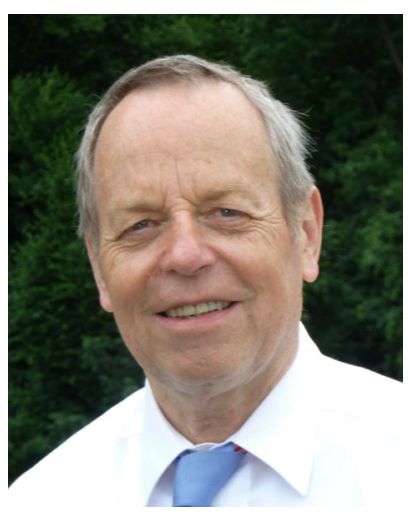

Em. O. Univ.-Prof. Dr. Günther Brauner
Das Europäische Forum Alpbach stellte im Jahr 2014 seine Technologiegespräche unter das Thema "Forschung und Innovation at the crossroad". Die Technische Universität Wien veranstaltete hierzu den Arbeitskreis 7 "Smart Energy: Herausforderungen an eine interdisziplinäre Zukunft".

Die wesentlichen Herausforderungen in der Energieversorgung werden in den nächsten Jahrzehnten durch den dramatischen Wandel von fossiler hin zu überwiegend erneuerbarer Energienutzung begründet. Die begrenzten Potenziale erneuerbarer Energiequellen und deren Fluktuation erfordern eine hohe Energieeffizienz und langfristige Speichertechnologien mit großen Kapazitäten. Bei der gesellschaftlichen Implementierung sind aber auch soziale Aspekte zu berücksichtigen. Basierend auf den interdisziplinären Inputs sollte im Arbeitskreis eine ökonomisch wie sozial gangbare Strategie für den gleichberechtig- ten Zugang zu sauberer Energie zu bezahlbaren Preisen entwickelt werden.

Da hierbei sehr komplexe Aufgabenstellungen zu lösen sind, werden Modellbildung und Simulation eine bedeutendere Rolle spielen. Die Energiewende betrifft die Städte mit all ihren Energieanwendungen, die Energieversorgung, die Industrie, das Gewerbe und alle Endnutzer von Energie. Die Energieforschung hat sich schon neu aufgestellt und sich von einer Komponentenforschung hin zur Systemforschung entwickelt.

Die technologischen Herausforderungen liegen in den großen installierten Leistungen, insbesondere von Wind und Photovoltaik, und den relativ kleinen Volllaststunden. Die Netze und Speichertechnologien müssen hierfür ausgebaut werden, und die dezentralen Systeme müssen versuchen, einen möglichst hohen Anteil der dort gewonnenen Energie durch flexiblere Energienutzung lokal zu verwenden.

Die Energiewende hat neben technologischen auch wirtschaftliche, politische und soziale Auswirkungen und erfordert längerfristig ein umsichtiges und systematisches Vorgehen. Sie betrifft alle Menschen. Deshalb wurden für die Gruppenarbeit des Arbeitskreises auch Studentinnen und Studenten aus allen österreichischen Universitäten und Fakultäten eingeladen, die mit Enthusiasmus und innovativen Ideen sehr interessante Lösungsvorschläge entwickeln konnten. Finden Sie im Anschluss zwei ausgewählte Beiträge der Alpbacher Technologiegespräche.
Brauner, Günther, Technische Universität Wien, Institut für Energiesysteme und Elektrische Antriebe, Gußhausstraße 25/370-1, 1040 Wien, Österreich (E-Mail: g.brauner@tuwien.ac.at) 